\title{
RAZONES PARA LA NO PRÁCTICA FÍSICA Y DEPORTIVA EN ADOLESCENTES DE LA REGIÓN DE ARAUCANÍA, CHILE
}

\section{REASONS FOR PHYSICAL AND SPORTS NON-PRACTICE IN ADOLESCENTS IN THE REGION OF ARAUCANÍA, CHILE}

\author{
Gerardo Fuentes-Vilugrón ${ }^{1}$ y Roberto Lagos-Hernández ${ }^{2}$ \\ gfuentes2019@alu.uct.cl; roberto.lagos@uautonoma.cl \\ ${ }^{1}$ Universidad Católica de Temuco, Chile \\ ${ }^{2}$ Universidad Autónoma de Chile, Chile
}

Envío Original: 2020-01-31 Reenviado: 2020-06-17, 2020-09-09 Aceptado: 2020-10-19

Publicado: 2020-11-12

Doi: https://doi.org/10.15517/pensarmov.v18i2.40531

\begin{abstract}
RESUMEN
La actividad física (AF) es importante para generar estilos de vida saludables. No obstante, se desconoce los motivos por los cuales las personas no mantienen hábitos saludables de AF. El objetivo de este estudio fue analizar los motivos para la no práctica de actividades físico-deportivas (AFD) en estudiantes entre los 11 y 19 años, en la región de Araucanía, Chile. A un total de 175 participantes se les aplicó Cuestionario para el Análisis de Motivación y la Práctica de la Actividad Físico-Deportiva. De 57 estudiantes que no practican AFD, 35 individuos que dejaron la práctica de actividad física señalan que se debe a la falta de tiempo y el segundo motivo es la falta de interés, en cuanto a los estudiantes que nunca han participado de AFD, 22 de ellos mencionan que el principal motivo para no practicar este tipo de actividades es que no se les da bien el deporte y manifiestan pereza y desgano. En conclusión, se hace muy necesario, tanto para profesores, monitores, o profesionales dedicados al área de la AF y la salud, entender a los procesos en que los adolescentes se encuentran, para hacer uso de sus motivaciones e incentivar la práctica de AFD. Puesto que, los adolescentes han cambiado drásticamente sus intereses y esas lógicas favorecen el alejamiento de las prácticas de AF.
\end{abstract}

Palabras clave: motivaciones, actividad física, actividad deportiva, Chile 


\begin{abstract}
Physical activity (PA) is important to generate healthy lifestyles. However, the reasons why people do not maintain healthy PA habits are unknown. The objective of this study was to analyze the reasons for not practicing physical-sports activities (AFD) in students between 11 and 19 years old, in the Araucanía region, Chile. A total of 175 participants were given a Questionnaire for the Analysis of Motivation and the Practice of Physical-Sports Activity. Of 57 students who do not practice AFD, 35 individuals who stopped practicing physical activity indicate that it is due to lack of time and the second reason is lack of interest, in terms of students who have never participated in AFD, 22 from they mention that the main reason for not practicing this type of activity is that they are not good at sports and show laziness and reluctance. In conclusion, it is very necessary, both for teachers, monitors, or professionals dedicated to the area of PA and health, to understand the processes in which adolescents find themselves, to make use of their motivations and encourage the practice of AFD. Since, adolescents have drastically changed their interests and these logics favor the distance from PA practices.
\end{abstract}

Key words: motivations, physical activity, sports activity, Chile

\title{
INTRODUCCIÓN
}

Esta investigación aborda las motivaciones que poseen los estudiantes de la región de la Araucanía para no practicar actividad físico-deportiva (AFD), entendiendo la importancia que implica este conjunto de acciones para la salud (Yáñez-Silva, Hespanhol, Gómez, y Cossio-Bolaños, 2014; López, Rodríguez, García, y Pérez, 2016) y considerando la relevancia de la etapa adolescente como un periodo crítico, debido a los cambios físicos, psicológicos y sociales que se evidencian (Gaete, 2015).

El rol de las AFD durante las etapas de crecimiento y desarrollo se hacen fundamentales, por su influencia y beneficios aportados al desarrollo físico, psicológico y social (Hidalgo-Rasmussen, Ramirez, e Hidalgo-San Martín, 2013). A pesar de los beneficios identificados de la práctica de la AFD, se observa que en el periodo de la adolescencia ha aumentado la inactividad física (IF) y las conductas sedentarias (CS), producto del uso de tecnologías entre otros factores. Además, se ha establecido que conductas sedentarias y falta de actividad física (AF) en los adolescentes están determinadas también por factores familiares (Lavielle, Pineda, Jáuregui, y Castillo, 2014), 
por tanto, si la familia no manifiesta interés por la práctica de actividad física, probablemente los hijos tampoco serán adeptos a estas acciones (Nuviala, Ruiz-Juan, y García, 2003).

Sin embargo, otras razones también influyen en la no practica de actividad física como por ejemplo no contar con referentes significativos que induzcan a la práctica de actividad física (Serra, Zaragoza, y Generelo, 2014), disponer de menos niveles de motivación autodeterminada (Mayorga-Vega, Parra, y Viciana, 2019), baja escolaridad (Escalante, 2011; Klee Oehlschlaeger, Tavares Pinheiro, Horta, Gelatti, y San'Tana, 2004) y tener condición de pobreza, condición que se ha estudiado, observando que el sedentarismo se correlaciona positivamente con niveles socioeconómicos más deprivados, reflejando que las familias pobres presentan un mayor sedentarismo en comparación con las familias no pobres (González, 2013). Sin embargo, otros estudios son contradictorios, por cuanto la actividad física se le asocia incluso a consumo de sustancias ilícitas. Finalmente, los adolescentes declaran que no hacen AF simplemente por falta de tiempo y ganas (Navas y Soriano, 2016).

En América Latina tres cuartos de la población tienen un estilo de vida sedentario, conductas que van en aumento con el pasar de los años (Vidarte-Claros, Vélez-Álvarez y Parra-Sánchez, 2012). El aumento del sedentarismo obliga a desarrollar hábitos de vida saludable a temprana edad para que perduren con el tiempo. En Chile, el sedentarismo alcanza un total de $89,4 \%$ según la Encuesta Nacional de Salud (Ministerio de Salud del Gobierno de Chile, 2017), entendiendo como conducta sedentaria a aquellas personas que no practican AF al menos 30 minutos seguidos, tres veces a la semana y fuera del horario laboral (Serón, Muñoz, y Lanas, 2010) esto, ha sido influenciado por cambios urbanísticos, tecnológicos, entre otros; lo que ha generado modificaciones importantes en los hábitos y estilo de vida de nuestra sociedad, fenómeno también asociado a sobrepeso, obesidad y enfermedades crónicas no transmisibles (Cristi-Montero y Rodríguez, 2014) debido a que las condiciones arquitectónicas, reducirían la actividad social (Bautista, Flores, y Guevara, 2018) y con ello las actividades motrices y recreativas asociadas a la AFD.

La IF y el sedentarismo se han convertido en uno de los problemas de la sociedad juvenil, tanto en la infancia, como también en la adolescencia (Bosque-Lorente y BaenaExtremera, 2014; Cuevas-Campos, García-Calvo, y Contreras, 2013; Martínez, et al., 2012) por lo tanto, es necesario entender que IF y sedentarismo no son lo mismo, a pesar de ser conceptos directamente relacionados. La conducta sedentaria es definida como la carencia de movimiento durante las horas de vigilia a lo largo del día, y es caracterizada por actividades que sobrepasan levemente el gasto energético basal (ver televisión, estar 
acostado o sentado), mientras que IF se define como el no cumplimiento de las recomendaciones mínimas internacionales de AF para la salud de la población (CristiMontero et al., 2015; Lalinde, Mayorga, y Cardona,_2014; Organiazación Mundial de la Salud [OMS], 23 de febrero, 2018).

Según la OMS la AF es definida como cualquier movimiento corporal producido por los músculos que resultan en gasto energético (Manzaneda, Lazo, y Málaga, 2015). La AF es un factor importante al momento de generar estilos de vida saludables y se considera además como un factor fundamental dentro de los procesos de promoción y prevención que deben implementar los profesionales de la Salud (Moreno y Cruz, 2015). La AF practicada con regularidad, tanto en adultos como en adolescentes, está asociada a menor riesgo de enfermedad cardiovascular, enfermedad coronaria y accidente vascular encefálico y sus efectos benéficos se han atribuido principalmente a la disminución del índice de masa corporal, menor probabilidad de padecer de resistencia a la insulina, favorece el aumento del colesterol HDL y mejoría de la hipertensión y función endotelial (Krämer et al., 2009; Arteaga, Bustos, Soto, Velasco, y Amigo, 2010; Cossío et al., 2017). Sin embargo, la AF, no solo aporta beneficios de tipo biológicos, sino también, de tipo psicológicos, demostrándose que se correlaciona negativamente con la depresión, ansiedad, victimización por los compañeros y una autoestima baja (Trejo, Jasso, Mollinedo, y Lugo, 2012).

Otro aspecto vinculado directamente a la AF es el ejercicio físico, entendido como una acción planificada cuyo objetivo es adquirir, mantener o mejorar la condición física (Escalante, 2011; Hernández y Licea, 2010), en este sentido, la práctica de ejercicio físico debe ser regulada en intensidad, frecuencia y duración, por lo que se considera como la mejor manera de mantener la capacidad funcional de las personas (Saz, Gálvez, Ortíz, y Saz, 2011). Sin embargo, se requiere de una sistematicidad y conducta regular, que podría ser una barrera a la adherencia para los adolescentes, quienes debido a la labilidad de sus conductas, podrían rehuir estas acciones por su carácter, rigor y disciplina, pese a que mejora la funcionalidad e impacta positivamente en el rendimiento académico, tareas cognitivas, atención selectiva y memoria a corto plazo (Fuentes, Lagos, Fernández y Fuentes, 2019; Riquelme, Sepúlveda, Muñoz, y Valenzuela, 2013) constituyéndose en un factor fundamental para la salud (Fuentes, 2009). En este mismo contexto, la actividad deportiva también posee un conjunto de beneficios psicobiológicos comprobados (Gómez, Daher, Vacirca, y Ciairano, 2012) y contribuye en el afianzamiento de conductas sociales 
favorecedoras del desarrollo afectivo, emocional y social (Fraile, 2010) por lo tanto su promoción colabora con el fomento de la adherencia a la AF para la adultez.

Es necesario esclarecer que, para efectos de este estudio, se usa el concepto de AFD como la unión de los tres términos enunciados ( $\mathrm{AF}$, ejercicio físico y actividad deportiva). A partir de estos antecedentes surge el cuestionamiento de por qué los estudiantes no practican AFD, lo que finalmente constituye el sustento desde dónde emerge el objetivo de esta investigación que consiste en analizar los motivos para la no práctica de AFD en estudiantes de séptimo a cuarto año medio, en la región de la Araucanía, que en este caso constituyó la segunda parte de un estudio mayor asociado a las motivaciones que manifiestan los escolares por desarrollar AFD.

\section{METODOLOGÍA}

\section{Diseño}

Para efectos de esta investigación se utilizará un enfoque cuantitativo, de corte transversal y con un diseño no experimental (Hernández, Fernández, y Baptista, 2006).

\section{Participantes}

La muestra está conformada por 57 estudiantes de Séptimo a Cuarto Año de enseñanza básica y media, con edades de 11 a 19 años, pertenecientes a 5 establecimientos educacionales de la Región de la Araucanía, distribuidos en las Comunas de Temuco, Padre Las Casas y Perquenco. El tipo de muestreo es por conveniencia, dado que los sujetos de estudio fueron escogidos dentro de una población mayor y por la facilidad en el reclutar de los estudiados (Mendieta, 2015). Cabe señalar que la presente investigación desglosó los datos de 57 estudiantes que manifestaron no practicar ningún tipo de AFD, a partir de los datos originales aplicados a 175 estudiantes. Puesto que, inicialmente se aplicó el cuestionario a 175 estudiantes, entre los que se diferenciaron aquellos que practican y no practican AFD (tabla 1), para posteriormente hacer uso de la muestra que no practican AFD para efectos de esta investigación.

\section{Instrumento}

El instrumento utilizado para la recolección de datos en esta investigación consiste en un único cuestionario para el análisis de Motivación y la Práctica de la Actividad FísicoDeportiva (CAMPAFD) de Hellín, Moreno y Rodríguez (2004). El cuestionario consta de 26 
apartados de carácter cerrado, organizado en 11 bloques relacionados entre sí, de los cuales se obtiene información sobre el comportamiento físico-deportivo de la población, el instrumento fue validado a través de diversos análisis de independencia entre variables mediante las pruebas de $X^{2}$ de Pearson completada con análisis de residuos (Hellín, Moreno, y Rodriguez, 2004). Además, este cuestionario se ha utilizado en diversos trabajos desde el 2004 a la fecha en distintos estudios vinculados a la motivación por la práctica de la actividad físico deportiva y constituye un referente en la materia en idioma español.

\section{Procedimiento}

La elección de las preguntas está orientada a la búsqueda de la variable de no práctica, por tanto las respuestas asociados a este ítem, fueros lo que dieron origen a los antecedentes que se presentan a continuación y que son parte de una investigación de mayor envergadura relacionadas con los motivos de práctica que manifiestan los estudiantes de enseñanza media de los establecimientos anteriormente indicados, referencias que están publicadas la investigación denominada Motivaciones hacia la práctica de actividad física-deportiva en estudiantes de La Araucanía (Fuentes y Lagos, $\underline{2019)}$.

A partir de los datos de los estudiantes que no practican ningún tipo de AFD, se hizo la presente indagación, como una estrategia de profundización de sus intereses, por tanto, los datos que aquí se muestran, constituye al estudio donde se enfatiza en el interés por conocer las razones que tienen los estudiantes para no practicar ningún tipo de AFD.

\section{Análisis de datos}

Para el procedimiento de análisis de los datos obtenidos a través del Cuestionario para el análisis de la motivación y la práctica de la actividad Físico-Deportiva, se hace uso de una planilla Microsoft Excel 2013@ para tener una organización preliminar de los datos y para el tratamiento y análisis estadístico de los datos obtenidos de tipo descriptivo comparativo, se hace uso del programa computacional SPSS @ versión 23.0.

La investigación contó con el consentimiento de los estudiantes y de sus padres y se llevó a cabo bajo los criterios deontológicos de la declaración de Singapur (Comisión nacional de Investigación Científica y Tecnológica [CONYCIT], 2013). 


\section{RESULTADOS}

El total de encuestados fue de 175 estudiantes con edades entre 11 a 19 años, con 115 sujetos de género masculino y 60 de género femenino.

Tabla 1

Práctica de Actividad Físico-Deportiva

\begin{tabular}{|c|c|c|c|c|c|}
\hline & & & \multicolumn{3}{|c|}{ Práctica de Actividad Físico- Deportiva } \\
\hline & & & Sí & No & Total \\
\hline & Masculino & $\mathrm{N}^{\circ}$ & 83 & 32 & 115 \\
\hline \multirow[t]{3}{*}{ Género } & & $\%$ & $47,4 \%$ & $18,3 \%$ & $65,7 \%$ \\
\hline & Femenino & $\mathrm{N}^{\circ}$ & 35 & 25 & 60 \\
\hline & & $\%$ & $20,0 \%$ & $14,3 \%$ & $34,3 \%$ \\
\hline \multirow[t]{2}{*}{ Total } & & $\mathrm{N}^{\circ}$ & 118 & 57 & 175 \\
\hline & & $\%$ & $67,4 \%$ & $32,6 \%$ & $100,0 \%$ \\
\hline
\end{tabular}

Fuente: Elaboración propia

Teniendo en consideración los objetivos de este estudio, en primera instancia se diferenciaron aquellos estudiantes que sí practicaban algún tipo de actividad físicodeportiva, en este caso 118 sujetos, equivalentes al 67,4\%, de aquellos que no practican ningún tipo de AFD, es decir 57 sujetos correspondientes al 32,6\% del total (Tabla 1).

De los 57 estudiantes que mencionan no practicar AFD, estos se dividen en dos grupos, como indica la Tabla 2, por un lado, están aquellos que nunca han realizado AF y los que por algún motivo abandonaron la AFD.

Tabla 2

Estudiantes que no practican Actividad Físico-Deportiva

\begin{tabular}{lccccc}
\hline & & Abandono & Nunca han realizado & Total \\
\hline \multirow{3}{*}{ Género } & Masculino & $\mathrm{N}^{\circ}$ & 23 & 17 & 40 \\
& & $\%$ & $40,4 \%$ & $29,8 \%$ & $70,2 \%$ \\
\cline { 2 - 6 } & Femenino & $\mathrm{N}^{\circ}$ & 12 & 5 & 17 \\
& $\%$ & $21,1 \%$ & $8,8 \%$ & $29,8 \%$ \\
\hline Total & $\mathrm{N}^{\circ}$ & 35 & 22 & 57 \\
& & $61,4 \%$ & $38,6 \%$ & $100,0 \%$ \\
\hline
\end{tabular}

Fuente: elaboración propia. 
Considerando los 22 estudiantes que nunca han realizado actividad física-deportiva, se evidenció que 14 estudiantes (63,6\%) no realizan AFD "Porque no se le da bien el deporte". De los 14 estudiantes que respondieron esta opción, 11 estudiantes (79\%) pertenecen al género masculino y 3 estudiantes (21\%) pertenecen al género femenino.

Considerando de igual manera estos 22 estudiantes que no realizan AFD, 11 indicaron que no son parte de estas actividades "Por pereza y desgano".

Por otro lado, de los 35 estudiantes que declararon haber abandonado la práctica de actividad físico-deportiva, 20 estudiantes $(57,1 \%)$ mencionaron que esto sucedió "Por falta de tiempo libre", pero adicionalmente y debido a que la opción de la encuesta permite dar más de una alternativa, se logró establecer que 19 estudiantes $(54,2 \%)$ abandonaron la práctica de actividad físico-deportiva "Porque dejó de interesarles", en esta opción, 14 estudiantes $(74 \%)$ pertenecían al género masculino, y 5 estudiantes (26\%) al género femenino.

\section{DISCUSION}

En cuanto a la ausencia de participación en AFD, se ha podido constatar que los motivos de no práctica de AFD se relacionan con el factor tiempo, y en otros casos las personas no le ven beneficios ni utilidad (Rodríguez-Romo, Boned-Pascual, y GarridoMuñoz, 2009), en esta misma línea de análisis, hay trabajos que han podido determinar que el índice de IF está presente mayoritariamente en las mujeres y la principal razón manifestada, es la falta de tiempo, en segundo lugar aparece la pereza (Rodríguez et al., 2013). Otros estudios también han sostenido que las condiciones de pobreza (González, 2013), la arquitectura y condiciones urbanisticas, constituyen agentes que no son promotores de las AF, así también el interés de la familia, constituye un importante promotor de interés por la AF (Nuviala et al., 2003), y la motivación autodeterminada (Mayorga-Vega et al., 2019; Moreno y Martínez, 2006), adicionalmente según antecedentes recopilados en investigaciones que han abordado esta situación, se ha podido constatar que el principal motivo de abandono de la práctica físico-deportiva, por ende, la no práctica, está condicionada por la participación de los encuestados en actividades académicas, y en segundo lugar la pereza, resultados que se asemejan a la presente investigación donde se pudo apreciar que el principal motivo de no práctica de AF es la falta de tiempo con un $57,1 \%$ de estudiantes entre hombres y mujeres, y en segundo lugar la falta de interés con un $54,2 \%$ de los estudiantes (Martínez et al., 2012). Al comparar los antecedentes de esta investigación con un estudio realizado en España llamado "Motivos de abandono de la 
práctica de actividad físico-deportiva en los estudiantes de bachillerato de la Provincia de Granada", se encontró que los resultados obtenidos son semejantes en ambas investigaciones, ya que en los dos casos, el principal motivo de la no práctica por abandono de la AFD es la falta de tiempo (Macarro, Romero, y Torres, 2010).

Desde otra perspectiva, en una investigación realizada el año 2005, se preguntó a los técnicos o entrenadores sobre el abandono de la práctica físico deportiva de adolescentes, los resultaron mostraron que la mayoría de los técnicos $(81,2 \%)$ dicen que el momento del abandono deportivo se produce cuando los deportistas pierden el interés por el deporte, lo que hace que la perspectiva dada por los técnicos se asemeje a los resultados obtenidos en esta investigación, la segunda causa principal de abandono, es la falta de interés por parte de los estudiantes (Nuviala y Nuviala, 2005).

Los antecedentes obtenidos en la presente investigación muestran que el principal motivo de los estudiantes para no practicar AF es la falta de tiempo, situación presente en el $57,1 \%$ del total de encuestados. Estos resultados son similares a los datos aportados en el año 2016 en un trabajo hecho con estudiantes chilenos, cuyas conclusiones señalan que el $31,5 \%$ de los estudiantes evaluados mencionan que los motivos para no practicar AF es porque "no tienen tiempo", y el segundo motivo más frecuente con un $29 \%$ es "por falta de ganas" (Navas y Soriano, 2016).

\section{CONCLUSIONES}

Se concluye que de los 57 estudiantes que no practican AFD, 35 sujetos señalan que se debe a la falta de tiempo, mientras que el segundo motivo que provoca el abandono de la práctica de AFD, es la falta de interés. En este mismo contexto, de los 22 sujetos que mencionan que nunca han practicado ningún tipo de AFD, se constató que el principal motivo de los estudiantes para no practicar este tipo de actividades es que no se les da bien el deporte y además manifiestan pereza y desgano. Los estudios que han abordado esta temática han llegado a conclusiones similares, por cuanto la ausencia de interés, la pereza o bien la sensación de que no es una actividad de su agrado, constituyen las principales situaciones que impiden la adherencia a las AFD. Ante esto, se sugiere profundizar en las motivaciones manifestadas por los estudiantes, con el fin de tener datos que arrojen información en esta materia. Además, se hace muy necesario, tanto para profesores, monitores, o profesionales dedicados al área de la AF y la salud, entender los procesos en que los adolescentes se encuentran, para hacer uso de sus motivaciones e incentivar la práctica de AFD, considerando intereses de los estudiantes y sus enfoques personales. En 
tal sentido, cobran relevancia los trabajos que estudian cómo las personas se implican de manera autónoma a las actividades que son representativas de sus intereses personales considerando los mecanismos psicológicos que condicionan sus conductas, por ejemplo observar qué acciones, juegos o deportes, suponen mayor adherencia o placer, qué tipo de actividades constituyen formas de acción motriz cuyo rol inclusivo reduce los riesgos de abandono, qué deportes son más aceptados, incluyendo aquellos no tradicionales que se alejan de los considerados hegemónicos, tradicionales o socialmente más aceptados, como es el caso del fútbol básquetbol o gimnasia, incluyendo otras manifestaciones que de manera emergente han ido ganando terreno como por ejemplo las actividades circenses, el parkour, la danza urbana o el Slackline en todas sus modalidades.

Las características más libres, menos competitivas y sus connotaciones liberales satisfacen muchas veces las demandas de movimientos, que se han ido perdiendo en el contexto de los programas de deporte escolar, actividades extracurriculares y las actividades deportivas incluidas en los planes y programas educativos establecidos. Con ello, se pretende una mayor orientación hacia la motivación autodeterminada, las investigaciones en esa línea muestran que dicha motivación está vinculada con la motivación intrínseca, mientras que la motivación extrínseca y sus dispositivos de regulación favorecen conductas no autodeterminadas e incluso caracterizadas por la falta de motivación, fenómenos observados en este estudio. Aparentemente los adolescentes, han cambiado drásticamente sus intereses y esas lógicas favorecen el alejamiento de las prácticas de AF cuando los recursos ofrecidos a nivel escolar y extraescolar no son atractivos, mermando drásticamente el interés, la motivación y el goce propio del movimiento, por tanto los profesionales están obligados a comenzar a estudiar otras manifestaciones de la motricidad humana, con la finalidad de adaptarse a los nuevos intereses juveniles, de lo contrario la tendencia hacia la inactividad física continuará en alza. La presente investigación posee como limitación el reducido número de estudiantes que fueron parte de la muestra, por lo tanto, se considera que sería un aporte, consolidar un número mayor de encuestados para hacer más robustas las conclusiones sobre que motiva a los estudiantes a no ser parte de acciones vinculadas a la AF. 


\section{REFERENCIAS}

Arteaga, A., Bustos, P., Soto, R., Velasco, N., y Amigo, H. (2010). Actividad física y su asociación con factores de riesgo cardiovascular. Un estudio en adultos jóvenes. Revista Médica de Chile, 138(10), 1209-1216. doi: https://doi.org/10.4067/S0034$\underline{98872010001100001}$

Bautista, J. M., Flores, M. L., y Guevara, M. L. (2018). Recuperación del espacio público para la reducción de la percepción de inseguridad: el caso del fraccionamiento Los Héroes en Puebla, México. Revista de Urbanismo, (39), 1-17. doi: https://doi.org/10.5354/0717-5051.2018.50489

Bosque-Lorente, M., y Baena-Extremera, A. (2014). Actividad físico-deportiva de los alumnos de tercer ciclo de primaria y de la Eso en su tiempo libre. Ágora para la Educación Física y el Deporte, (16), 36-49. Recuperado de http://agorarevista.blogs.uva.es/files/2014/05/agora 16 1c bosque y baena.pdf

Comisión Nacional de Investigación Científica y Tecnológica (CONYCIT). (2013). Declaración de Singapur sobre la integridad en la investigación. Recuperado de https://www.conicyt.cl/fondap/files/2014/12/DECLARACI\%C3\%93N-SINGAPUR.pdf

Cossío, M., Méndez, J., Luarte, C. L., Vargas, R., Canqui, B., y Gómez, R. (2017). Patrones de Actividad Física de Adolscentes escolares: Validez, confiabilidad y propuesta de percentiles para su evaluación. Revista Chilena de Pediatría, 88(1), 73-82. doi: https://doi.org/10.1016/..rchipe.2016.07.010

Cristi-Montero, C. C., y Rodríguez, F. (2014). Paradoja: "activo físicamente pero sedentario, sedentario pero activo físicamente". Nuevos antecedentes, implicaciones en la salud y recomendaciones. Revista Médica de Chile, 142(1), 72-78. doi: http://dx.doi.org/10.4067/S0034-98872014000100011

Cristi-Montero, C., Celis-Morales, C., Ramírez-Campillo, R., Aguilar-Farías, N., Álvarez, C., y Rodríguez-Rodríguez, F. (2015). ¡Sedentarismo e inactividad física no son lo mismo!: una actualización de conceptos orientada a la prescripción del ejercicio físico para la salud. Revista Médica de Chile, 143(8), 1089-1090. doi: https://doi.org/10.4067/S0034-98872015000800021

Cuevas-Campos, R., García-Calvo, T., y Contreras, O. (2013). Perfiles motivacionales en Educación Física: una aproximación desde la teoría de las Metas de Logro 2x2. Anales de Psicología, 29(3), 685-692. doi: http://dx.doi.org/10.6018/analesps.29.3.175821 
Escalante, Y. (2011). Actividad física, ejercicio físico y condición física en el ámbito de la salud pública. Revista Española de Salud Pública, 84(4), 325-328. Recuperado de http://scielo.isciii.es/scielo.php?script=sci arttextypid=S1135-57272011000400001

Fraile, A. (2010). El desarrollo moral en el deporte escolar en el contexto Europeo: Un estudio basado en dilemas sociomorales. Estudios Pedagógicos, 36(2), 83-97. doi: https://doi.org/10.4067/S0718-07052010000200005

Fuentes, F. J. (2009). El deporte en el marco de la Educación Física. Sevilla, España: Wanceulen Editorial Deportiva.

Fuentes Vilugrón, G., y Lagos Hernández, R. (2019). Motivaciones hacia la práctica de actividad físico-deportiva en estudiantes de la Araucanía. Revista Ciencias de la Actividad Física UCM, 20(2), 1-13. doi: https://doi.org/10.29035/rcaf.20.2.3

Fuentes, M., Lagos, R., Fernández, W., y Fuentes, G. (2019). Análisis del Programa Vida Sana y el significado que subyace al discurso de estudiantes de Lautaro, Chile. Journal of Sport Pedagogy and Research, 5(3), 28-35. Recuperado de http://www.ipg.pt/scpd/files/JSPR SE 53 2019.pdf

Gaete, V. (2015). Desarrollo psicosocial del adolescente. Revista Chilena de Pediatría, 86(6), 436-443. doi: https://doi.org/10.1016/j.rchipe.2015.07.005

Gómez, N., Daher, S., Vacirca, F., y Ciairano, S. (2012). Relación de la Actividad Deportiva, el Estrés, la Depresión, la importancia a la Salud y el uso de Alcohol en los Adolescentes Bolivianos. Revista de Investigación Psicológica, (7), 85-92. Recuperado de http://www.scielo.org.bo/pdf/rip/n7/n7a06.pdf

González, W. J. (2013). Aspectos socioeconómicos y familiares asociados en niños y adolescentes obesos. Revista de Ciencias Sociales, 19(1), 120-130. Recuperado de https://www.redalyc.org/pdf/280/28026467016.pdf

Hellín, P., Moreno, J., y Rodríguez, P. (2004). Motivos de práctica físico-deportiva en la región de Murcia. Cuadernos de Psicología del Deporte, 4(1-2), 101-115. Recuperado de https://revistas.um.es/cpd/article/view/112481/106721

Hernández, J., y Licea, M. E. (2010). Papel del ejercicio físico en las personas con Diabetes Mellitus. Revista Cubana de Endocrinología, 21(2), 182-201. Recuperado de http://scielo.sld.cu/scielo.php?script=sci arttext\&pid=S1561-29532010000200006

Hernández, R., Fernández, C., y Baptista, P. (2006). Metodología de la investigación. México: McGraw-Hill.

Hidalgo-Rasmussen, C. A., Ramírez-López, G., y Hidalgo-San Martín, A. (2013). Actividad Física, conductas sedentarias y calidad de vida en adolescentes universitarios de 
Ciudad Guzmán, Jalisco, México. Ciência \& Saúde Coletiva, 18(7), 1943-1952. doi: https://doi.org/10.1590/S1413-81232013000700009

Klee Oehlschlaeger, M. E., Tavares Pinheiro, R., Horta, B., Gelatti, C., y San'Tana, P. (2004). Prevalência e fatores associados ao sedentarismo em adolescentes de área urbana. Revista de Saúde Pública, 38(2), 157-163. Recuperado de https://www.scielosp.org/pdf/rsp/2004.v38n2/157-163/pt

Krämer, V., Acevedo, M., Orellana, L., Chamorro, G., Corbalán, R., Bustamante, M. J., ... Navarrete, C. (2009). Actividad física y potencia aeróbica: ¿Como influyen sobre los factores de riesgo cardiovascular clásicos y emergentes? Revista Médica de Chile, 137(6), 737-745. doi: https://doi.org/10.4067/S0034-98872009000600002

Lalinde, P. C., Mayorga, J. M., y Cardona, W. D. (2014). Realación entre la Actividad Física, el Sedentarismo y la calidad seminal. Revista Chilena de Obstetricia y Ginecología, 79(4), 323-329. doi: http://dx.doi.org/10.4067/S0717-75262014000400012

Lavielle, P., Pineda, V., Jáuregui, O., y Castillo, M. (2014). Actividad Física y Sedentarismo: Determinantes Sociodemográficos, familiares y su impacto en la Salud del Adolescente. Revista de Salud Pública, 16(2), 161-172. doi: https://doi.org/10.15446/rsap.v16n2.33329

López, F. J., Rodríguez, P. L., García, E., y Pérez, J. J. (2016). Relación entre la práctica físico-deportiva y el consumo de alcohol en adolescentes escolarizados de Murcia (España). Archivos Argentinos de Pediatría, 114(2), 101-106. doi: https://doi.org/10.5546/aap.2016.101

Macarro, J., Romero, C., y Torres, J. (2010). Motivos de abandono de la práctica de actividad físico-deportiva en los estudiantes de Bachillerato de la Provincia de Granada. Revista de Educación, (353), 495-519. Recuperado de https://dialnet.unirioja.es/servlet/articulo?codigo=3288034

Manzaneda, A. J., Lazo-Porras, M., y Málaga, G. (2015). Actividad física en pacientes ambulatorios con diabetes mellitus 2 de un Hospital Nacional del Perú. Revista Peruana de Medicina Experimental y Salud Pública, 32(2), 311-315. Recuperado de http://www.scielo.org.pe/scielo.php?script=sci arttext\&pid=S172646342015000200016

Martínez, A. C., Chillón, P., Martín-Matillas, M., Pérez, I., Castillo, R., Zapatera, B., ... Delgado-Fernández, M. (2012). Motivos de abandono y no práctica de actividad físicodeportiva en adolescentes españoles: estudio Avena. Cuadernos de Psicología del 
Deporte

12(1),

45-54.

Recuperado

de

http://scielo.isciii.es/scielo.php?script=sci arttext\&pid=S1578-84232012000100005

Mayorga-Vega, D., Parra Saldías, M., y Viciana, J. (2019). Condición física, actividad física, conducta sedentaria y predictores psicológicos en adolescentes chilenos: diferencias por género. Cultura, Ciencia y Deporte, 14(2), 233-241. Recuperado de https://ccd.ucam.edu/index.php/revista/article/view/1337

Mendieta, G. (2015). Informantes y Muestreo en investigación Cualitativa. Investigaciones Andina, 17(30), 1148-1150. Recuperado de http://www.redalyc.org/pdf/2390/239035878001.pdf

Ministerio de Salud del Gobierno de Chile. (2017). Encuesta Nacional de Salud 2016-2017: primeros resultados. Recuperado de https://www.minsal.cl/wpcontent/uploads/2017/11/ENS-2016-17 PRIMEROS-RESULTADOS.pdf

Moreno, J. A., y Martínez, A. (2006). Importancia de la teoría de la autodeterminación en la práctica físico-deportiva: Fundamentos e implicaciones prácticas. Cuadernos de Psicología del Deporte, 6(2). Recuperado de https://revistas.um.es/cpd/article/view/113871

Moreno, J. E., y Cruz, H. F. (2015). Percepción de la Actividad física en estudiantes de enfermería frente a fisioterapia. Enfermería Global, 14(4), 192-200. doi: https://doi.org/10.6018/eglobal.14.4.209811

Navas, L., y Soriano, J. (2016). Análisis de los motivos para practicar o no, actividades físicas extracurriculares y su relación con el autoconcepto físico en estudiantes chilenos. Revista iberoamericana de Psicología del Ejercicio y del Deporte, 11(1), 6976. doi: http://hdl.handle.net/10045/52609

Nuviala, A., y Nuviala, R. (2005). Abandono y continuidad de la práctica deportiva escolar organizada desde la perspectiva de los técnicos de una Comarca Aragonesa. Revista Internacional de Medicina y Ciencias de la Actividad Física y el Deporte, 5(20), 295307. doi: https://doi.org/10486/3654

Nuviala, A., Ruiz-Juan, F., y García, M. E. (2003). Tiempo libre, ocio y actividad física en los adolescentes. La influencia de los padres. Retos: Nuevas tendencias en Educación Física, Deportes y Recreación, (6), 13-20. doi: https://doi.org/10.47197/retos.v0i6.35078

Organización Mundial de la Salud (OMS). (23 de febrero, 2018). Actividad Física. Recuperado de https://www.who.int/es/news-room/fact-sheets/detail/physical-activity 
Riquelme, D., Sepúlveda, C., Muñoz, M., y Valenzuela, M. (2013). Ejercicio Físico y su influencia en los procesos cognitivos. Revista Motricidad y Persona: serie de estudios, (13), 69-74.

Recuperado

de https://dialnet.unirioja.es/servlet/articulo?codigo=4736022

Rodríguez, F., Palma, X., Romo, Á., Escobar, D., Aragú, B., Espinoza, L., ... Gálvez, J. (2013). Hábitos alimentarios, actividad física y nivel socioeconómico en estudiantes Universitarios de Chile. Nutrición Hospitalaria, 28(2), 447-455. doi: https://doi.org/10.3305/nh.2013.28.2.6230

Rodríguez-Romo, G., Boned-Pascual, C., y Garrido-Muñoz, M. (2009). Motivos y barreras para hacer ejercicio y practicar deportes en Madrid. Revista Panamericana de Salud Pública, 26(3), 244-254. doi: https://doi.org/10.1590/S1020-49892009000900009

Saz, P., Gálvez, J. J., Ortiz, M., y Saz, S. (2011). Ejercicio Físico. Medicina Naturista, 5(1), 18-23. Recuperado de https://dialnet.unirioja.es/servlet/articulo?codigo=3401250

Serón, P., Muñoz, S., y Lanas, F. (2010). Nivel de Actividad física a través del Cuestionario Internacional de Actividad Física en población Chilena. Revista Médica de Chile, 138(10), 1232-1239. doi: https://doi.org/10.4067/S0034-98872010001100004

Serra, J., Zaragoza, J., y Generelo, E. (2014). Influences from "significant others" for physical activity practice in teenegers. Revista Internacional de Medicina y Ciencias de la Actividad Física y el Deporte, 14(56), 735-753. Recuperado de https://repositorio.uam.es/bitstream/handle/10486/663005/RIMCAFD56 10 eng.pdf ?sequence $=4$ \&isAllowed $=\mathrm{y}$

Trejo, P. M., Jasso, S., Mollinedo, F. E., y Lugo, L. G. (2012). Relación entre la actividad física y obesidad en escolares. Revista Cubana de Medicina General Integral, 28(1), 34-41. Recuperado de http://scielo.sld.cu/scielo.php?script=sci arttext\&pid=S086421252012000100005

Vidarte-Claros, J. A., Vélez-Álvarez, C., y Parra-Sánchez, J. H. (2012). Niveles de sedentarismo en población de 18 a 60 años. Manizales, Colombia. Revista de Salud Pública, 14(3), 415-426. Recuperado de https://revistas.unal.edu.co/index.php/revsaludpublica/article/view/27867/39779

Yáñez-Silva, A., Hespanhol, J. E., Gómez, R., y Cossio-Bolaños, M. (2014). Valoración de la Actividad Física en adolescentes escolares por medio de Cuestionario. Revista Chilena de Nutrición, 41(4), 360-366. doi: https://doi.org/10.4067/S0717$\underline{75182014000400003}$ 